\title{
Film-based and digital-based holographic and shearographic interferometry: an educator's perspective
}

Huai Shang

Huai Min Shang, "Film-based and digital-based holographic and shearographic interferometry: an educator's perspective," Proc. SPIE 4588, Seventh International Conference on Education and Training in Optics and Photonics, (28 May 2002); doi: 10.1117/12.468705

SPIE Event: Education and Training in Optics and Photonics 2001, 2001, Singapore, Singapore 


\title{
Film-Based and Digital-Based Holographic and Shearographic Interferometry - An Educator's Perspective
}

\author{
H.M. Shang \\ Department of Mechanical Engineering, National University of Singapore, \\ 10 Kent Ridge Crescent, Singapore 119260 \\ Email: mpeshm@nus.edu.sg
}

\begin{abstract}
Since the advent of the laser, the rapid development of new interferometric techniques such as holography and shearography has brought precision measurement and non-destructive testing to a new dimension. Many novel variations of these techniques are developed and used, but in general, their working principles are based on the comparison between two optical gratings - the known reference grating and the object grating that is distorted by the test surface. Comparison of the two gratings will result in the formation of a fringe pattern that depicts lines of equal spatial coordinates and surface displacements (both in-plane and out-of-plane), and lines of equal surface slopes and surface displacement gradients.

Conventionally, optical interferometry is developed using high-resolution films but with the rapid advancement of computer and image-processing technology, these film-based techniques have given way to digital techniques. Whilst the theories of both film-based and digital techniques are now well developed, students, however, often find it difficult to understand how the fringe pattern is formed and reconstructed, especially when they shift from the film-based to the digital techniques. In this paper, the formation of the optical gratings (that is, the object grating and the reference grating) is explained in the light of the well-known Young's interference fringes. The formation and reconstruction of the visible fringe pattern is explained in the light of the well-known moiré phenomenon caused by the interference of the two optical gratings. In the film-based techniques, the visible fringe pattern is explained with reference to image-addition (that is, addition of two optical gratings) and subsequent image-multiplication (that is, multiplication of two optical gratings). In the digital techniques, the fringe pattern is reconstructed using image-addition and subsequent image-subtraction.
\end{abstract}

Keywords: holography, shearography, optical interferometry, optical grating

\section{INTRODUCTION}

Arising from the invention of the laser, new optical techniques such as double-exposure holography and shearography have been developed; existing techniques such as photoelasticity, projected grating and moiré have also been reexamined and re-developed for better precision ${ }^{[1-7]}$. The underlying principles of these methods are based on comparing the optical grating that is distorted by the test surface with the known reference optical grating. The comparison, which is made through interference of the two gratings, will result in the formation of interference fringes-lines that depict lines of equal spatial coordinates and surface displacements (both in-plane and out-of-plane), and lines of equal surface slopes and surface displacement gradients. Traditionally, optical interferometry is achieved using high-resolution photographic films, but with the rapid advancement of computer and image-processing technology, optical interferometry is now frequently carried out digitally.

Although the theories of optical interferometric techniques are now well-developed, students have often found it difficult to understand how the fringe pattern is formed and reconstructed especially when they progress from film-based to digital-based techniques. In this paper, an attempt is made to explain the principles of these techniques in the light of two well-known phenomena, namely, light interference of two point-sources (formation of Young's interference fringes) and light interference of two optical gratings (formation of Moiré fringes). With this perspective, the various film-based and digital-based optical methods can be easily understood. 


\section{REVIEW OF YOUNG'S INTERFERENCE FRINGES AND MOIRÉ PATTERNS}

When two beams of light that are generated from the same coherent light source interfere, the intensity distribution $I$ recorded either on the film or on the image plane of a CCD camera may be described mathematically as follows.

$$
I=A+B \cos \phi
$$

where $A$ and $B$ are optical constants, and $\phi$ is the phase-difference between the two point-light sources at any point on the image plane. The intensity variation due to the phase-difference manifests as a fringe pattern known as the Young's interference fringes, an optical grating that is described mathematically by the equation of a hyperbolid ${ }^{[8]}$. Thus, depending on the relative position between the point-light sources and the screen, the interference fringes may appear as straight lines, full circles or circular segments.

It is worth noting that the density of the Young's fringes will increase with the distance between the two point-light sources, and that the resolution of the recording media determines the maximum density in which the fringe-lines can be recorded. Thus, as the resolution of a high-resolution film is very much higher than that of a CCD camera (which is used in digital techniques), the distance between the two point-light sources can be much larger in film-based techniques than in digital-based techniques.

When the records of two slightly mismatched high-density optical gratings are stacked and viewed against a strong light source, a low-density optical pattern known as the Moiré fringe pattern is formed. Thus, if the intensity distributions $I_{l}$ and $I_{2}$ of the optical gratings are each described in the same manner as in Eq. (1), the resulting intensity distribution $I_{T}$ is given by the following expression.

$$
I_{T}=I_{1} I_{2}=A^{2}+\frac{B^{2}}{2} \cos \left(\phi_{2}-\phi_{1}\right)+\left[A B \cos \phi_{1}+A B \cos \phi_{2}+\frac{B^{2}}{2} \cos \left(\phi_{1}+\phi_{2}\right)\right]
$$

The two gratings are high frequency waveforms, hence the square-bracketted terms in Eq. (2) cannot be resolved by the human eye and are nullified. Using the relation $\left(\phi_{2}-\phi_{1}\right)=\Delta$, Eq. (2) becomes

$$
I_{T}=A^{2}+\frac{B^{2}}{2} \cos \Delta
$$

Thus, the reconstructed visible fringes are known as moiré fringes that are related to the phase-change $\Delta$ between the two gratings of intensity distributions $I_{I}$ and $I_{2}$.

Suppose the intensity distributions $I_{1}$ and $I_{2}$ of the optical gratings are each recorded using a CCD camera that is linked to a frame grabber and a computer. It is also permissible to take the difference of $I_{1}$ and $I_{2}$, a software-driven imageprocessing technique known as image-subtraction. Thus, from Eq. (1) and using the relation $\left(\phi_{2}-\phi_{1}\right)=\Delta$, imagesubtraction yields the following expression for the resulting intensity $I_{T}$.

$$
\left|I_{T}\right|=\left|I_{2}-I_{1}\right|=\left|-2 B \sin \left(\frac{\phi_{1}+\phi_{2}}{2}\right) \sin \left(\frac{\Delta}{2}\right)\right|
$$

Again, a visible fringe pattern similar in form as the moiré pattern (Eq. (3)) will be reconstructed on the computer monitor through image-subtraction.

\section{FILM-BASED DOUBLE-EXPOSURE HOLOGRAPHY AND SHEAROGRAPHY}


Double-exposure holography ${ }^{[1-4]}$ and double-exposure shearography ${ }^{[5-7]}$ were developed independently using the laser as the light source. In double-exposure holography, a reference beam and an object beam are brought to interfere at the recording film, one exposure made before and another after the object is deformed slightly. A holographic fringe pattern depicting lines of constant-displacements is reconstructed by illuminating the doubly exposed recording film with the reference beam. In shearography, however, the use of an image-shearing device can be likened as attaching the lightsource for the reference beam on the object surface. Therefore, aside from the manner in which the fringe pattern is reconstructed, shearography can be perceived as a technique that encompasses double-exposure holography - the use of a large image-shearing device gives rise to displacement-related holographic fringes whereas the use of a small imageshearing device leads to the formation of strain-related shearographic fringes ${ }^{[9]}$.

In the case of double-exposure holography and double-exposure shearography, the resulting intensity distribution on a doubly exposed photographic film, where the phase-change $\Delta$ is encoded, is the superposition of the intensities $I_{1}$ and $I_{2}$ of the two optical gratings; this is also known as image addition. Thus, the following expression is obtained.

$$
I_{T}=I_{1}+I_{2}=2\left[A+B \cos \left(\frac{\phi_{1}+\phi_{2}}{2}\right) \cos \left(\frac{\Delta}{2}\right)\right]
$$

where $\Delta=\left(\phi_{2}-\phi_{1}\right)$ is the phase-change between the two exposures. Thus, the reconstructed fringe-lines are related to $\frac{\Delta}{2}$. It is worth noting that to reconstruct holographic fringe-lines ${ }^{[1-4]}$, the doubly exposed hologram is to be illuminated with the same reference beam. To reconstruct shearographic fringe-lines from a doubly exposed shearogram, a high-pass Fourier filtering optical device must be used as otherwise, the fringe visibility is grossly reduced ${ }^{[10]}$.

\section{DIGITAL-BASED DOUBLE-EXPOSURE HOLOGRAPHY AND SHEAROGRAPHY}

The intensity equation (Equation 1) is still applicable in digital holography and shearography. Thus, the use of imagesubtraction (Equation 4) enables reconstruction of a fringe pattern that is related to the phase-change $\Delta$. This phasechange may be caused by deformation of the illuminated test surface.

\section{RELATIONSHIP BETWEEN PHASE-CHANGE AND PHYSICAL QUANTITIES}

In double-exposure holography and double-exposure shearography, the phase-change $\Delta$ between two recordings may be caused by an incremental change $(\delta \lambda)$ in the wavelength, an incremental change $(\delta n)$ in the refractive index, and an incremental change $(\delta L)$ in the geometrical path length in the following manner.

$$
\Delta=-\frac{2 \pi L n}{\lambda^{2}}(\delta \lambda)+\frac{2 \pi L}{\lambda}(\delta n)+\frac{2 \pi n}{\lambda}(\delta L)
$$

Through Eq. (6), the well-known methods of double-wavelength, double-refractive index, two point-source, and carrierfringe for surface profiling and deformation measurement are developed. ${ }^{[1-3]}$

\section{DETERMINATION OF PHASE-CHANGE USING DIGITAL TECHNIQUES}

In the determination of phase-change $\Delta$ using digital techniques, fast Fourier transform, phase stepping or multiple-frame phase shifting are used together with a phase unwrapping algorithm to account for $2 \pi$ phase jumps. The need for phase unwrapping arises because the calculated phase-change $\Delta$ is wrapped within the interval $[-\pi,+\pi]$. As an illustration, Figure 1 shows a typical shearographic fringe pattern of a plate subjected to an incremental point load between two exposures. 


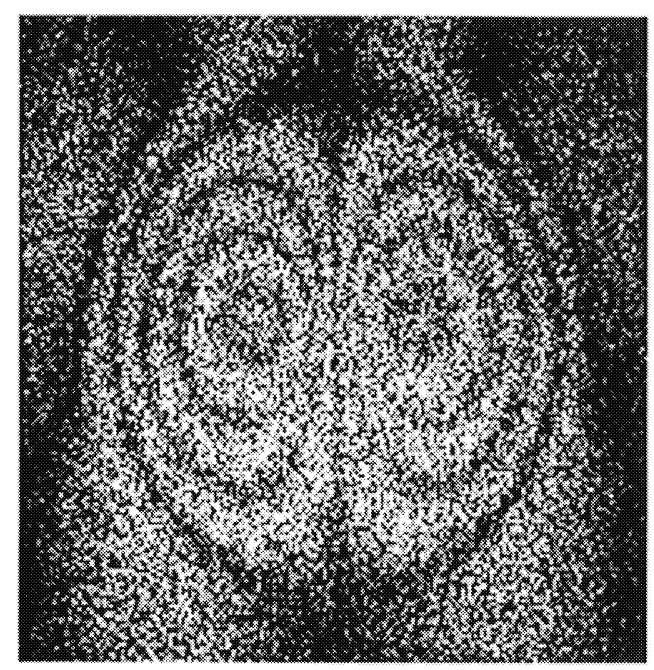

Fig. 1. Typical shearographic fringe pattern of a plate under central point load.

\section{CONCLUSION}

In the early days when high-resolution photographic films were used, reconstruction of the visible fringe pattern is explained in the light of image-addition (that is, addition of two optical gratings) and subsequent image-multiplication (that is, multiplication of two optical gratings). With the rapid advancement of computer and image processing techniques, the tedious film-based techniques have quickly given way to high-speed digital techniques in which the fringe pattern can be reconstructed within fractions of a second using image-addition and subsequent image-subtraction.

In this paper, the formation of the optical gratings (that is, the object grating and the reference grating) is explained in the light of the well-known Young's interference fringes. The formation and reconstruction of the visible fringe pattern is explained in the light of the interference of the two optical gratings. This approach show alleviate difficulty faced by students in understanding how fringe patterns are formed and reconstructed, especially when they are required to shift from the conventional to the digital techniques.

\section{REFERENCES}

[1] Erf RK. (1974) Holographic Nondestructive Testing, New York: Academic Press.

[2] Vest CM. (1979) Holographic Interferometry, New York: John Wiley.

[3] Jones R \& Wykes C. (1989) Holographic and Speckle Interferometry: A Discussion Of The Theory, Practice And Application Of The Techniques, UK: Cambridge University Press.

[4] Abramson N. (1981) The Making and Evaluation of Holograms, Academic Press, New York.

[5] Leendertz JA \& Butters JN. (1973) An image-shearing speckle pattern interferometer for measuring bending moments. J. Phys. E: Sci. Instrum., 6, 1107-1110.

[6] Hung YY. (1974) A speckle-shearing interferometer. Optical Communications, 11, 132-135.

[7] Hung YY. (1982) Shearography: a new optical method for strain measurement and nondestructive testing. Optical Engineering, 21(3) 391-395.

[8] Shang HM, Quan C, Tay CJ \& Hung YY. (2000) Generation of carrier fringes in holography and shearography. Applied Optics, 39(16):2638-2645.

[9] Shang HM, Hung YY, Luo WD \& Chen F. (2000) Surface profiling using shearography. Optical Engineering (Special Issue), 139(1) 23-31.

[10] Toh SL, Tay CJ, Shang HM \& Lin QY. (1993) Analysis of shearogram reconstruction. Applied Optics, 32(25), 4929-4933. 